\title{
IMPORTANCIA DE LA CULTURA EMPRESARIAL
}

\author{
IMPORTANCE OF CORPORATE CULTURE \\ LUIS BORTESI LONGHI * \\ Docente Asociado de la Facultad de Ciencias Contables - UNMSM \\ [Recepción: Agosto de 2011 / Conformidad: Octubre de 2011]
}

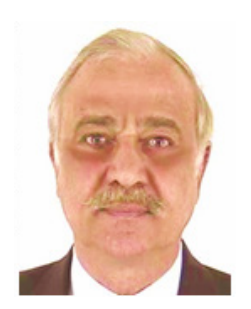

\section{Resumen}

La cultura empresarial tiene dos direcciones, una hacia el exterior que apunta al entorno social, a la comunidad e indirectamente también al país entero por la contribución de la empresa al empleo y al desarrollo nacional. Asimismo la cultura empresarial comprende de manera importante y prioritaria el cuidado del ambiente y todo ese conjunto que se acaba de describir obedece al título general de "Responsabilidad Social". La otra dirección se orienta hacia el interior de la organización y en ese sentido se refiere a mantener la armonía y la solidaridad como valores empresariales que constituyen un poderoso Activo Intangible. Es notable la importancia que han cobrado progresivamente los activos intangibles que en el pasado se circunscribían a las marcas y patentes pero que en la actualidad se han extendido a otros valores sutiles de trascendencia fundamental a tal punto que a veces constituyen el rubro más importante del patrimonio empresarial.

En el presente artículo nos ocuparemos particularmente de analizar de manera pormenorizada los factores que se consideran remarcables en materia de cultura empresarial al interior de la organización.

Palabras clave: Organización - Cultura Valores - Intangibles.

\section{Abstract}

The corporate culture has two addresses, one pointing outward to the social, community and indirectly also the whole country by the company contribution to employment and national development. Corporate culture also comprises an important and primary environmental care and all that joint described above reflects the general title of "Social Responsibility". The other direction is toward the inside of the organization and in this sense refers to maintaining harmony and solidarity as company values that are a powerful Intangible Assets. It is remarkable that have become increasingly important intangible assets in the past were limited to trademarks and patents but now have spread to other values fundamental importance subtle to the point that sometimes constitute the largest item of heritage business. In this article we will particularly discuss in detail the factors considered remarkable in terms of corporate culture within the organization.

Key words: Organization - Culture - Values - Intangibles.

\footnotetext{
* Doctor en Ciencias Económicas - PUCP, Magister en Ciencias Económicas - PUCP, Docente Investigador de la Facultad de Ciencias Contables - UNMSM, Docente Asociado de la Facultad de Ciencias Contables - UNMSM.

E-mail: luisbortesil@hotmail.com
} 


\section{INTRODUCCIÓN}

En general, el vocablo cultura deriva de "cultivo" en el sentido de explorar, alentar y perfeccionar el ejercicio y conocimiento de los valores más estimables de la existencia. La cultura entonces está consustancialmente vinculada a la moral en el sentido de priorizar y mantener fidelidad a los valores tales como la justicia, la verdad, el bien y la belleza.

Es cierto que no existe una cultura única y universal puesto que en varias etapas de la historia de la humanidad así como en las diversas latitudes regionales el concepto de cultura no es idéntico. Si se la vincula a la religión resulta claro lo que se acaba de señalar y en ese sentido se distingue el pensamiento oriental del occidental. El hinduismo y el budismo son fuentes representantes de la cultura oriental y tienen un cierto paralelo de correspondencia con la tradición que nos es más cercana es decir la corriente Judeo Cristiana - Islámica.

Sin embargo la perspectiva religiosa no es la única que ilustra la riqueza cultural toda vez que existe por separado el arte y la ciencia. La política también es en su sentido más profundo un hecho cultural así como las relaciones sociales e incluso todas las costumbres y tradiciones.

Una distinción importante que conviene hacer cuando se habla de cultura es que no hay que confundirla con erudición. Este error se comete con mucha frecuencia y con mayor medida cuando interviene la tecnología la que, en rigor, no se identifica con la cultura sino más bien con las aplicaciones prácticas y el dominio de la materia y de la energía. La erudición se manifiesta por ser un conocimiento de carácter pasivo y acumulativo y su principal destreza consiste en la buena memoria. Por ejemplo se puede ser erudito como crítico cinematográfico o como cono- cedor de la historia universal. Por lo general la persona erudita tiene la vocación de la lectura y posee considerables bibliotecas sobre la materia que lo atrae aunque no es la creatividad su principal fruto. En cambio la cultura que, tal como se señaló, consiste en el cultivo de valores está vinculada a los conocimientos abstractos y creativos.

La información que abunda profusamente en nuestros días, vía internet por ejemplo, no presupone necesariamente un acervo cultural porque los datos y las estadísticas no garantizan el conocimiento y en algunas ocasiones pueden hasta confundirlo; en cambio entendida en buenos términos la cultura tiene que ver con la sabiduría y ésta no se apoya necesariamente en la información y los datos.

Dicho lo anterior se puede colegir que la cultura debe servir para vivir mejor en el sentido que el cuidado y respeto por los valores más estimables de la existencia humana deben permitir una mejor calidad de vida y una existencia más comprensiva.

Conviene refrescar el concepto de cultura y revisar los verdaderos alcances de la tan presumida y vanagloriada "era del conocimiento". Porque la verdad es que si se reemplaza la abstracción y el cultivo de los valores como son el bien, la verdad, la belleza, la justicia y la paz interior, por el culto a las imágenes y se abandona la introversión por el divertimiento, la dirección en la que discurre la humanidad es inferior. En efecto, la "diversión", literalmente hablando, consiste en proyectar la conciencia hacia el exterior y en consecuencia su resultado es exactamente contrario a la vitalidad interior que se experimenta en las profundidades de la meditación donde se desarrolla literalmente el "entusiasmo", la vitalidad interior del espíritu y es éste un hecho indiscutible.

De otro lado, y paralelamente, hay que insistir en que hay una gran diferencia entre 
información y conocimiento. La información es de carácter general, vulgar, no selectiva y muchas veces caótica. El conocimiento es otra cosa. La información tiene que ver con los datos y las estadísticas y con el acervo de registros, el conocimiento tiene que ver con la sabiduría.

El entorno social, el paisaje, el ambiente de trabajo, la satisfacción de las necesidades, el trabajo vocacional y creativo, el ocio productivo, la buena salud integral, el sentido del humor, la sensación de libertad, la Fe, han sido los factores que se han cotejado para ser analizados en un contexto donde sin embargo habrán de prevalecer dos que son la salud y la Fe.

Ahora bien, como fruto y resultado de una excelente calidad de vida se comprueba, en síntesis, un estado de armonía que se manifiesta en la paz interior y en la alegría de vivir siendo su exponente más claro el de la virtud de la generosidad que consiste en la satisfacción de dar antes que recibir.

Lo antedicho se puede resumir con un brillante texto de San Pablo en su epístola a los Gálatas, V - 22/23: "los frutos del espíritu son caridad, gozo, paz, paciencia, benignidad, bondad, longanimidad, mansedumbre, fe, modestia, continencia, castidad. Para los que viven de esta suerte no hay ley que sea contra ellos".

Para concluir, haremos un paralelo entre la cultura y la inteligencia en el sentido que son dos capacidades que se potencian mutuamente porque de lo contrario habría un defecto en los conceptos. Es muy difícil definir lo que es la inteligencia humana porque es una noción muy rica y existen diversas interpretaciones y explicaciones sobre ella, a mayor razón en tiempos actuales donde cobra importancia la evaluación de la inteligencia emocional. Sin embargo, las respuestas más frecuentes para referirse a la inteligencia son que se trata de la capacidad para resolver problemas o también, en ocasiones, se dice que la inteligencia es la capacidad de adaptación. Pero ambas respuestas son extensivas también al reino animal y no serían privativas del género humano.

La inteligencia humana es la capacidad para comprender las causas de las cosas y, por sobre todo, comprender al prójimo y visto así tal facultad no sería aplicable al reino animal. Y es precisamente en esa dirección donde la cultura y la inteligencia se potencian pues de lo contrario tanto la inteligencia como la cultura, sin aplicación útil, no valdrían la pena.

\section{De la Cultura Empresarial al Interior de la Empresa}

Una empresa es una organización humana que combina recursos y los administra con el propósito principal de obtener un excedente económico. Los recursos en cuestión son los siguientes:

Personal
Tecnológicos
Financieros
Naturales
Investigación
El tiempo

A su vez, la clasificación general de activos es la siguiente: activos físicos, activos financieros y activos intangibles. El tema de los intangibles ha venido enriqueciéndose y perfeccionándose últimamente hasta alcanzar una importancia de primera prioridad porque los activos físicos vienen a corresponder, si se permite la analogía, al "cuerpo" los activos financieros a la fuerza y la energía y los intangibles representan "el alma" de la organización. Se explican como activos o recursos invisibles que pertenecen al dominio de la psicología y el espíritu y le confieren a la organización un notable valor patrimonial 
porque en definitiva los intangibles son una ventaja en cuestión de competitividad y en este sentido es obvio que es particularmente en el área del personal donde se puede ubicar los intangibles más preciosos y alcanzar la virtud del holismo que quiere decir que el conjunto o el todo es más que la suma de las partes. Un buen ejemplo es el de una orquesta sinfónica donde, gracias a la batuta de un excelente director, se extrae una música de conjunto que transmite una belleza superior a lo que ejecutara cada instrumentista por su cuenta.

El trabajo en equipo representa uno de los aspectos más importantes en la institución y se puede comparar con el ejemplo anterior de la orquestación para aumentar la producción y mejorar la productividad. No cabe duda que la figura del líder, como individuo, es clave en este mismo contexto, pero no hay que confundir "don de mando" con "liderazgo". Es la misma diferencia que se puede establecer entre los conceptos de autoridad y poder. La primera es un hecho moral de fe y credibilidad en la persona del líder al cual siguen no porque sea obligatorio sino porque tiene una especie de imán que atrae $\mathrm{e}$ inspira confianza, en cambio lo segundo, es decir el poder, deriva de la fuerza, de la sumisión y del temor. Lo que tiene más relieve en el tema de la orquestación y el holismo es que el personal trabaje con seguridad y con alegría condiciones que seguramente mejorarán la productividad y son un intangible de lo más valioso. En cambio si en la atmósfera laboral hay tensión, miedo, frustración, recelos y chismes el pronóstico de esa empresa es malo.

\section{Aspectos sensibles en la organización empresarial}

Motivación al personal.- En este aspecto lo más importante es que el trabajador, por humilde y modesto que sea su puesto, se sienta útil y esté convencido de que pertenece a un conjunto de ganadores y no de derrotados. El más grave error en que puede incurrir un jefe de personal es el de amonestar a alguien espetándole a viva voz y al borde del insulto que “ $¡$ está harto de él porque no sirve para nada!" porque seguramente la frustración de dicha persona será irreversible, a manera de una lesión. En la organización empresarial (en general en cualquier conjunto humano, incluyendo la familia) mucho más sagaz es el estímulo que el castigo y el hecho de que el personal trabaje estimulado conviene a todos, tanto los individuos como el conjunto y a los propios accionistas.

El reclutamiento.- Incorporar nuevo personal en la organización implica siempre un riesgo que en ocasiones puede resultar fatal. Habitualmente el procedimiento radica en la evaluación del currículum vitae del postulante y en las "referencias" personales que eventualmente aporte el candidato. La práctica ha demostrado que dicho tamiz es insuficiente porque, independientemente de los galardones académicos que obren en favor del ingresante, tan importante como dichos datos, o aún más, lo que será más valioso y lo profundo que se debe analizar es el perfil psicológico, el carácter y las cualidades bondadosas de la persona. Puede darse el caso, no poco frecuente, en que desde el punto de vista objetivo y profesional el currículum acuse una trayectoria académica brillante incluyendo estudios en el extranjero y el dominio de idiomas y sin embargo dicho individuo sea un Judas disfrazado que al comienzo actúe como un cordero pero que con el correr de algún tiempo se convierta en un verdadero demonio disociador. Esta es la razón por la cual es indispensable contar con un equipo de psicólogos de primer orden que desempeñen su trabajo en el proceso de reclutamiento. 
Evaluación del personal.- Con toda seguridad la profesión más delicada es la del Juez puesto que es muy difícil juzgar con imparcialidad y ponderación ya que naturalmente intervienen la simpatía o la antipatía y muy probablemente el subjetivismo.

Existen dos preguntas elementales para satisfacer la justicia en el proceso de evaluación del personal: la primera es ¿quién evalúa a quien? Y la segunda cuestión consiste en la tablilla que contiene los conceptos y los puntajes. De primera impresión puede parecer absurda la pregunta de quién evalúa a quien porque la respuesta lógica sería que el superior evalúa al subalterno, sin embargo la experiencia demuestra que el jefe no siempre es necesariamente el que se encuentra en las condiciones más completas para calificar al personal puesto que la evaluación vertical puede ser sustituida por otra de carácter horizontal, en el equipo con el mismo rango. Lo que sí es muy probable es que el gerente consigne una muy buena nota a su simpática secretaria.

Una incógnita que permanece vigente es la que está encerrada en la acreditación en el sentido que, por ejemplo al asignar una calificación ISO se puede interponer la duda de quién califica al calificador al igual que quién capacita al capacitador. La prueba que avala esta aserción está en el hecho de que las tres clasificadoras de riesgo internacionales son corresponsables de la crisis financiera internacional toda vez que calificaron como excelentes a los bonos hipotecarios "subprime" que causaron la catástrofe financiera internacional.

Con respecto a la plantilla con los puntajes y los conceptos a ser evaluados también hay reservas conceptuales por lo relativo que pueden ser las respuestas. Por ejemplo, con respecto al tema de la puntualidad se estima que es puntual aquel que marca en horario su correspondiente ingreso al trabajo, pero puede ser que dicho personaje "puntual" una vez que ingresa va a la cafetería a leer el periódico. Otro tema crítico es el de juzgar si el empleado evaluado tiene capacidad para trabajar en equipo. Depende, muchas veces, de cuán atractivo resulta ser el famoso equipo puesto que en este caso interviene la simpatía y la homogeneidad del grupo. Si existen incompatibilidades insuperables resulta prácticamente imposible trabajar en equipo.

En resumen, la evaluación al personal es un asunto extremadamente delicado que en balance de pros y contras puede resultar nocivo para la organización. Lo más importante en una institución avanzada y compacta es la autoevaluación en el sentido que, en todos los casos, el personal acepte y suscriba los resultados no con el afán de sanción o amonestación sino con el objeto de superar los eventuales defectos que se le señalen.

La precariedad.- Lo precario, es decir lo inestable o que dura poco, siempre es atentatorio contra la salud institucional porque cualquier esfuerzo puede ser desvirtuado por lo efímero. Si se está cambiando a cada rato el manual de organización y funciones, los métodos, las políticas, los planes y los objetivos no se puede afianzar la cultura empresarial y la organización competitiva y excelente. Es verdad, sin embargo, que en nuestro mundo contemporáneo las condiciones, sobre todo técnicas, cambian vertiginosamente y por lo tanto es obvio que hay que estar preparado para el cambio, pero al referirnos a la precariedad no estamos apuntando a eso, sino a la estabilidad de ánimo al interior de la empresa, en el sentido de los valores inmutables que impulsan el espíritu de la organización. Lo contrario sería algo así como que un político un día se declara socialista, al día siguien- 
te comunista y pasado mañana capitalista. $\mathrm{O}$ como si una sociedad fuese católica durante el verano, agnóstica durante el otoño y atea en el invierno. $\mathrm{Y}$ en este sentido hay que recusar de plano el tan cacareado "pragmatismo" que, en pocas palabras, significa "yo no me caso con nadie" porque no asumo ningún compromiso doctrinario, de principios, de premisas, de valores culturales inamovibles. Política de remuneraciones.- Este no es un aspecto sensible sino más bien "hipersensible" porque existe la tendencia vulgar de asumir que el monto salarial implica cuantitativamente lo el trabajador vale lo cual es evidentemente imposible puesto que cada individuo es un universo. Una norma básica que se debe cumplir es que a igualdad de rango tiene que haber equivalencia en la retribución porque a veces se comete el error de establecer escalas absurdas como decir jefe de departamento a, b, c, o también diseñar categorías para gerentes.

Las normas que rigen la política salarial deben ser claras y transparentes evitando pagos adicionales disfrazados "por lo bajo" con el método que se conoce con el mote de "planilla negra" porque eso se presta obviamente a suspicacias que desmoralizan a la gente que actúa de buena fe. Asimismo lo ideal en materia de remuneraciones es que no exista una abismal diferencia de sueldos en la pirámide organizacional. Vale la pena mencionar también que es un error e incluso una vulgaridad establecer un comedor para empleados y otro "para obreros" o durante la época de Navidad repartir canastas de diferentes precios.

En una empresa que se inspira en la cultura, cuyo principal valor es la solidaridad y por lo tanto está impulsada por el esfuerzo del trabajo colectivo, el personal debe participar en las utilidades en proporciones razonables y esa política conviene a todos, incluyendo los accionistas, porque con dicho incentivo lo más probable es que aumente la productividad y por lo tanto las utilidades. Claro está que el requisito para una participación justa es que el contador no haga trucos en el balance con el mezquino propósito de ocultar la verdad.

La rutina.- En cualquier actividad humana la rutina es un enemigo oculto que a manera de una lenta enfermedad va progresivamente atrofiando los sentidos y la lucidez. En cualquier actividad humana, incluyendo por ejemplo los ritos de piedad como asistir a la Santa Misa como una costumbre automática sin prestar mayor atención ni concentración a la devoción y al mensaje del Evangelio del día.

No todos los trabajos, sin embargo, están expuestos en la misma medida al peligro de la rutina puesto que aquellos que dependen exclusivamente de la creatividad, como es el caso del artista, la automatización y esa especie de robotización que perfilan los rasgos de la rutina no aparecen. En cambio en el caso extremo opuesto como es el del ascensorista la pérdida de la conciencia de lo que se está haciendo es prácticamente inevitable y este ejemplo puede hacerse extensivo a toda labor que se hace por repetición donde abundan actividades en el mundo contemporáneo como son los videojuegos que pervierten la capacidad reflexiva de los niños y en general se puede hablar también del auge que ha cobrado la famosa "cultura de la imagen".

Una profesión que está sumamente expuesta a la mediocridad de la rutina es la del contador si es que se acostumbra siguiendo habitualmente su plan de cuentas y haciendo los registros al mismo ritmo y utilizando el software y llegar al extremo de ser convertido casi en una máquina más. Por eso se dice, y con toda razón, que el contador verdaderamente creativo es el que interpreta los datos 
y recomienda medidas preventivas, a manera del médico, actitud lúcida que no concede el menor lugar a la rutina. Pero es bueno decir que la del contador es una de las profesiones que están expuestas a la automatización y por supuesto que no es la única ya que la verdad es que la mayoría de las actividades pueden convertirse en insípidas y aburridas. A propósito de lo que se ha dicho es pertinente referirse a la reingeniería que tiene precisamente como uno de sus objetivos principales estimular la creatividad y un estado de conciencia lúcido, despierto y alerta. En pocas palabras la reingeniería es una técnica reflexiva y psicológica que consiste en un autollamado permanente, como un test, donde se pregunta lo siguiente: primero, ¿qué estoy haciendo?, segundo, ¿para qué lo estoy haciendo, es decir para qué sirve? $\mathrm{Y}$ tercero ¿lo puedo hacer mejor?

La intriga.- Los chismes, las habladurías y los rumores que crecen como bola de nieve alentados por la morbosidad de algunas personas proclives a la cháchara y la conversación frívola son siempre nocivos en cualquier organización institucional porque fomentan el escándalo y generalmente atentan contra el prestigio de personas honestas. Los documentos que circulan subrepticiamente bajo el escudo cobarde del anonimato forman parte de este problema y deben ser desterrados radicalmente y rechazados en público. Cuando comienza a circular un rumor con el clásico estribillo "dicen que ....." el director de recursos humanos debe convocar a la totalidad del personal y en asamblea pública aclarar las cosas o bien desmentirlas así como el sol derrite un copo de nieve. En una organización que posee la suficiente base en materia de cultura nunca habrá lugar para la conducta innoble.

Funciones y atribuciones.- En el Manual de Organización y Funciones, además del co- rrespondiente organigrama, se enuncian en modo gramatical infinitivo las competencias que corresponden a cada repartición y por lo tanto es obvio que ese manual es clave para un funcionamiento eficiente de la institución y la experiencia demuestra que a menudo dicho documento no es lo suficientemente claro y operativo.

En primer lugar se debe decir que el enunciado de responsabilidades no debe ser innecesariamente extenso, es decir no debe exceder de cinco o seis puntos ya que es una mala señal querer hacer el manual como si se tratara de un código de derecho penal. Lo que suele ocurrir es que se solicita al propio personaje que proponga el enunciado de sus funciones $y$ entonces dicho funcionario instintivamente formula el mayor número posible de las funciones que él mismo habrá de desempeñar impulsado por el afán de demostrar la importancia de su puesto y esta actitud configura un error.

En segundo lugar tenemos el peligro de las superposiciones en el sentido que no está lo suficientemente bien delimitado el terreno que abarca cada repartición. Este defecto es mucho más común de lo que a primera vista pudiera parecer y deriva en el caos administrativo ya sea porque dos, o más, departamentos superponen sus tareas causando confusión, o también porque a la hora de la evaluación de resultados, en caso de fracaso, se acusan mutuamente de no haber cumplido los objetivos.

Del otro lado puede aparecer una especie de conflicto de intereses entre dos áreas que tienden a cumplir su cometido. Por ejemplo en el caso de la cartera de clientes donde el gerente comercial vende pero no es el área encargada de cobranzas y generalmente vende al crédito porque su meta es vender lo más posible. El gerente financiero, al comprobar que un cliente, por bueno que haya 
sido, está incurriendo en una morosidad peligrosa para la empresa decide cortarle el crédito y entonces el gerente comercial se enoja alegando que dicho cliente es predilecto. En este escenario el gerente financiero lleva claramente la razón e incluso puede optar por remitir la cartera impaga a la asesoría jurídica con el objeto de iniciar cobranza coactiva.

Y en tercer lugar está el problema de las atribuciones. Si se adjudica una responsabilidad a un determinado departamento es obligatorio que paralelamente se le asignen los recursos, atribuciones y la capacidad de decisión correspondientes para que pueda cumplirla porque lo contrario además de ser una injusticia acarreará seguramente un fracaso total. Sería algo así como acudir donde un sastre, entregarle un metro de tela y encargarle que haga un abrigo o un terno con saco pantalón y chaleco.

El gerente preponderante.- Este punto tiene relación con el anterior aunque se trata de un aspecto más específico. A nivel horizontal no es aconsejable que uno de los gerentes sea "el que más pesa" porque eso puede trabar la fluidez en el desempeño de los demás y sin embargo es muy frecuente que el gerente financiero, porque maneja la plata, tenga virtualmente subordinados a los otros gerentes cuando se trata de agilizar un desembolso como por ejemplo los viáticos para un viaje o la compra de los boletos de avión y en este caso se ha visto que los funcionarios, aún teniendo rango de gerentes, tienen que perder su tiempo para estar rogándole al encumbrado gerente financiero que por favor los atienda. Además de configurar una injusticia que atenta contra la dignidad de los colegas, la cultura empresarial debe predominar con paridad, con espíritu de colaboración, reciprocidad y respeto mutuo.

Si se observa el asunto detenidamente se puede comprobar que también a nivel nacional en el Poder Ejecutivo acontece lo mismo puesto que es evidente que por lo general, en materia de desembolsos, prácticamente todos los ministros dependen del Ministro de Economía y Finanzas.

El presupuesto debe ser aprobado regularmente, es decir de manera reglamentaria, pero una vez aprobado cada repartición debe tener autonomía y agilidad para cumplir sus tareas y alcanzar sus objetivos y sus metas. Por eso es importante que durante la formulación y discusión presupuestaria se calcule el costo unitario de las metas y entonces las prioridades queden marcadas y los compromisos y responsabilidades, así también como las atribuciones sean indiscutibles.

El auditor interno.- En el cuadro jerárquico el auditor interno debe reportar al Directorio y no al gerente general puesto que su labor de control abarca también a la gerencia general y por lo tanto no debe estarle subordinado. En principio y hablando en términos generales hay que precisar que si bien la función del auditor concierne principalmente al examen de los registros contables no se circunscribe exclusivamente a esto y puede extenderse a la evaluación y control de la organización, los métodos, los objetivos y los sistemas.

Debe entenderse que el principal propósito de la auditoría no es castigar sino proponer medidas correctivas para mejorar las cosas aunque claro está que si se comprueba una infracción es imperativo sancionar y si se trata de un delito procede enviar a la cárcel al delincuente. Sin embargo lo importante está en el hecho de que el auditor, que es el encargado del alto control, no actúe nunca prepotentemente sembrando el miedo y la tensión, sospechando siempre de todo y de todos, lleno de prejuicios infundados. Un auditor que actúa como un ogro es inconveniente en la organización.

Es muy frecuente que el auditor interno colisione con el contador por ejemplo cuando toma la iniciativa de proceder a un arqueo 
de caja intempestivamente con el ánimo "de atrapar al ladrón”, y cuando hace observaciones enojosas pero en el fondo intrascendentes. Lo importante en el contexto de una correcta cultura empresarial es que el auditor ayude al contador ya que seguramente tiene la experiencia suficiente porque por lo general los auditores son profesionales maduros. Capacitación permanente.- Las destrezas y las técnicas administrativas se encuentran en constante evolución por lo que resulta imprescindible la actualización permanente y la iniciativa para investigar y estudiar debe ser impulsada tanto por el individuo cuanto por la institución. Si la empresa le propone a un empleado que tiene buen pronóstico otorgarle una beca de estudios el desembolso que origina dicha decisión no es un gasto sino un activo intangible. Está supuesto que la persona favorecida por dicha beca tendrá que suscribir un compromiso contractual de permanencia cuando termine sus estudios pero también resulta lógico que al cabo de los mismos, si ha demostrado un buen aprovechamiento, sea premiado con un incremento en sus remuneraciones.

En cuanto a la capacitación resulta particularmente ventajoso que se manejen los idiomas extranjeros y en el caso de nuestro país el inglés, en primer lugar, pero también el portugués, son idiomas útiles que exaltan la calificación profesional y ese mérito también debe ser reconocido. La alusión a la lengua portuguesa se hace en virtud de las perspectivas que el Perú tiene con su gigantesco vecino, el Brasil, país con el cual se habrá de incrementar notablemente el intercambio impulsado, entre otras cosas, por la carretera interoceánica.

En lo relativo a la iniciativa personal para estudiar e investigar no es lo más recomendable circunscribirse exclusivamente a la Internet porque no siempre la información y el material que proporciona la Internet es confiable y de excelente calidad. Es por lo tanto indispensable que se recurra a la mejor bibliografía y a partir de las consultas buscar un asesoramiento en las personas de prestigio en la materia. Es una tarea ardua pero tiene su compensación y proporciona mayor solvencia, seguridad y aplomo en sí mismo. Si la empresa, en el entendido de practicar una cultura organizacional que prefiere el trabajo en equipo, advierte que un empleado está evolucionando y que aporta cada vez mejores ideas comprobará que se trata de un esfuerzo individual plausible que naturalmente tiene que ser reconocido. Lo ideal es que los interesados se matriculen en diplomados, maestrías y doctorados en la Universidad, aunque acá es menester establecer una advertencia: no es honesto buscar el camino más fácil e inscribirse en las instituciones superiores que demuestran la mayor laxitud y relajo otorgando doctorados a diestra y siniestra animadas por sobre todo en sostener el más alto presupuesto de ingresos posible. La gente debe comprender que no se trata de obtener "el cartón" sino de aprender de verdad y tener la plena conciencia de que el diploma logrado resulta de un mérito profesional y académico verdadero. Una conducta contraria configuraría una estafa y un engaño tanto para sí mismo como para el centro de trabajo.

Con respecto a la actualización, tal vez sea pertinente referirnos a un caso concreto que está en el centro de nuestra profesión. Se trata de la contabilidad electrónica (y también al comercio electrónico) con cuya técnica se han reemplazado los viejos libros contables y se han modificado los registros lo que obliga naturalmente a los contadores a investigar y aprender a manejar esta nueva técnica que probablemente no estudiaron cuando se licenciaron, porque es muy reciente.

Trabajos a presión.- ¡iMuy urgente!! ¡iRush!! Son las expresiones orales y los proveídos 
que se emiten como costumbre en las empresas mal organizadas donde se produce un clima de crisis permanente y se trabaja en un ambiente de delirio que se llega a parecer al de un manicomio. La velocidad y la agilidad son virtudes aconsejables pero la manía de la prisa por la prisa es una enfermedad psicológica que casi siempre afecta los resultados. Es un hecho indiscutible que en toda sociedad se presentan situaciones de emergencia que imponen una reacción apurada como si se tratara de un terremoto o de un incendio $y$ en tal circunstancia por supuesto que hay que correr a manejar el riesgo, es más, se debe tener estrategias contingentes para que en la eventualidad de una emergencia se esté preparado para afrontarla, pero esta eventualidad debe ser la excepción y no la regla. Para ilustrar la diferencia pongamos el siguiente escenario: viernes por la tarde, llama el gerente de área, convoca un grupo de trabajo $y$ ordena que se prepare un informe que ha solicitado el directorio. Entonces el jefe del equipo le pregunta al gerente cuándo hay que entregar el informe y obtiene la siguiente respuesta de un hombre con los ojos desorbitados e inflamados: ¡cómo que para cuándo! ¡i Para ayer!! Es casi seguro que el esfuerzo que realizará ese grupo, sacrificando el fin de semana, salga mal y en consecuencia dicho trabajo haya que rehacerlo una o más veces porque la experiencia demuestra que por lo general los trabajos hechos a presión no son de calidad.

Lo anterior de ninguna manera quiere decir que se avance a paso de tortuga y en un ambiente de molicie, de pereza y relajamiento, situación que sería peor que la anterior, sino con el adecuado ritmo, porque hay que reconocer que todas las cosas obedecen a un orden (que quiere decir un ritmo) y tienen un ciclo de maduración. Sin embargo en numerosas organizaciones se fomenta lo que se llama "el manejo del estrés" de gente neurótica que vive exaltada y confunde la agilidad y la velocidad con la precipitación y la desesperación.

En una empresa que fomenta la cultura se trabaja "con clase" y con ahínco, con agilidad, con concentración, con serenidad y es la puntualidad uno de los valores más importantes en una organización que posee cultura respetando por sobre todo el tiempo de los demás y por supuesto cumpliendo con rigor los horarios de las citas aunque éstas fueran acordadas con personal subalterno y humilde. Desafortunadamente en nuestro país muy frecuentemente se comprueba lo contrario y esta es una de las causas del subdesarrollo.

Un caso concreto que atenta contra la dignidad humana es la cultura del "fast food" que significa que hay que comer rápido sin ni siquiera lavarse las manos, para ganar tiempo, y lo que esta conducta acarrea es que las víctimas de esa otra manía contraen úlceras $y$ gastritis y tienen que tomar pastillas para poder dormir.

Familiares en la organización.- Por lo general la presencia y la incorporación de familiares constituye un riesgo en el sentido de confundir los aspectos funcionales y las categorías jerárquicas con los supuestos afectos y obligaciones que se deben por razón de los vínculos de parentesco. Entonces lo que ocurre es que se suscitan discusiones en la casa y se superpone el tema de la empresa al de la celebración que sería lo normal ese día domingo en el domicilio. Se podría tal vez plantear un punto de vista contrario y argumentar que naturalmente los familiares son de mayor confianza pero eso no es necesariamente cierto puesto que también cabe la posibilidad de que los parientes procuren medrar y sacar provecho propio asumiendo subjetivamente una prerrogativa tan sólo por el hecho 
de la relación familiar. El riesgo que se ha señalado se comprueba de manera palmaria en los partidos políticos donde con frecuencia la presencia de los familiares lejos de contribuir a la causa representa un obstáculo y un entorpecimiento en el discurrir de los planteamientos y ejecución programática.

Los "seres queridos" y las queridas al interior de la empresa configuran un serio problema que se advierte con suma frecuencia cuando se establecen lazos pasionales que desbordan la normalidad y provocan tensión y desequilibrio en la organización. La cultura empresarial no consiente ese tipo de manifestaciones como son los celos y las pasiones que deprimen la armonía y la buena marcha. Conclusiones.-

La empresa demuestra el grado de su cultura en dos direcciones: una hacia el exterior referida a la comunidad, al entorno y al cuidado del medio ambiente y la otra hacia el interior con respecto al grado de cultivo de los valores más estimables de la existencia humana. La consolidación de la armonía, la solidaridad, la lealtad y en general un agradable ambiente de trabajo aumentan la productividad tanto cuantitativa como cualitativamente.
La virtud de la cultura empresarial constituye un activo muy importante que se clasifica en "intangibles".

Los intangibles son activos inmateriales que confieren a la organización una ventaja competitiva porque perfeccionan los resultados.

\section{REFERENCIAS BIBLIOGRÁFICOS}

1. Agullo Tomás

“Trabajo, Individuo y Sociedad"

Editorial Pirámide

2. Girón María Eugenia

"Secretos de Lujo"

Editorial Empresarial S.L.

3. Guénon René

"Autoridad Espiritual y Poder Temporal"

Editorial Paidós

4. Hampton David R.

"Administración"

Mc Graw-Hill

5. San Pablo

"Epístola a los Gálatas"

Santa Biblia 